\title{
Politische Macht und richterliche Gewalt: Das portugiesische Modell
}

\section{Die Verunglimpfung der Dritten Gewalt als Bedrohung der Demokratie}

\section{Eine europäische Geschichte}

In für die italienische Justiz schwierigen Zeiten hat der Präsident der Republik, Pertini, als Vorsitzender einer Sitzung des Kontrollgremiums für die Justiz am 23. Juli 1981 folgendes geäußert: »Autonomie und Unabhängigkeit der Justiz sind wertvoll, denn sie sind als Voraussetzungen für die Implementierung aller Prinzipien und Vorschriften der Verfassung zu betrachten«. Die richterliche Unabhängigkeit war immer der gordische Knoten für diejenigen, die sie kontrollieren wollten. Sie ist kein politisch neutrales Konzept, sondern ist eng mit aktuellen sozialpolitischen Standpunkten innerhalb des politischen Systems verbunden und unterliegt dem Zeitgeist. 1994 begann der Herausgeber der Zeitung »Le Monde« sein den Beziehungen zwischen politischer Macht und Justiz gewidmetes Editorial mit einem ironischen Einleitungssatz, der berühmt wurde: »Ein Gespenst geht in der Republik um, das Gespenst des >Richterstaates « . Dieses Gespenst - als Bedrohung der Demokratie - wird heraufbeschworen, wenn immer die Justiz mit Gruppen oder Personen in Berührung kommt, die der Welt der Politik oder der Wirtschaft zuzurechnen sind.

In der Tat wurde die Verfolgung des »Richterstaates«, der jeglicher demokratischer Legitimierung entbehrte, zum ersten Male im Jahre 1911 in den Vereinigten Staaten von Amerika heraufbeschworen. Die Gründe für diese Verfolgung unterscheiden sich ganz bedeutend von denen, die heutzutage zu derselben Beschuldigung führen. Zu jener Zeit wurde das Oberste Bundesgericht von den amerikanischen progressiven Senatoren aufgrund der Tatsache angegriffen, dass das Gericht häufig solche Staats- und Bundesgesetze für verfassungswidrig erklärte, die tendenziell die Ausübung wirtschaftlicher Freiheiten zu kontrollieren suchten. Diese Streitigkeit stammte aus dem Jahre 1886 als das Oberste Bundesgericht erstmals unter Verwendung einer extensiven Interpretation des 14. Verfassungszusatzes, der ursprünglich der schwarzen Bevölkerungsgruppe das Recht auf die amerikanische Staatsbürgerschaft zugestand, die Gesetze einiger Staaten für verfassungswidrig erklärte, die die Beschränkung der Macht großer Kapitalgesellschaften zum Ziel hatte. Das führte schließlich zu einer völligen Umkehr der Rechtsprechung, die nun die mächtigsten Bürger schützte. 1932, nach der Großen Depression, waren die Sozialgesetze bezüglich des »New Deal« von Roosevelt durch das Oberste Bundesgericht verhindert worden. Arthur Schlesinger sprach im Zusammenhang mit dem »New Deal« von einer wahren politischen Gegenoffensive der Richter, die gegen die Regierungspolitik dieser Zeit waren. Die Positionen des Obersten Bundesgerichts führten daher - als politische Konsequenz - zur Zustimmung des (demokratischen) Präsidenten, die Organisation des Gerichts zu reformieren: die Erhöhung der Anzahl von Richtern sollte die traditionellen liberalen Strömungen auflösen. Den amerikanischen Richtern wurde vorgeworfen, den wirtschaftlichen Macht- 
habern zu nahe zu stehen und sich den Prinzipien des orthodoxen Liberalismus zu unterwerfen, was wiederum die demokratische Politik konterkarierte.

Heutzutage ist der Vorwurf des »Richterstaates « in der Neuen und Alten Welt immer noch weit verbreitet, aber die ursprüngliche Bedeutung wurde in ihr Gegenteil verkehrt. In den Vereinigten Staaten bewegte die von der Regierung nach den Vorfällen vom 11. September eingeführte außerordentliche Einschränkung der bürgerlichen Rechte (heimliche Festnahmen, Militärgerichte, Anhörungsgeheimhaltung, vorläufige Festnahmen auf unbestimmte Zeit, die Aufhebung des Rechts des Angeklagten auf einen Rechtsanwalt seines Vertrauens) einige Bundesgerichte dazu, das Justizministerium zur Offenlegung von Informationen über die Festgenommenen zu zwingen - entgegen den Vorkehrungen der Exekutive. Auch hier hat die Entscheidung der Richter, die zu Verteidigern der verfassungsmäßigen Freiheiten berufen wurden, der politischen Macht Grenzen, Hindernisse oder teilweise Behinderungen bereitet.

Während der letzten Jahrzehnte traten die häufigsten Konflikte zwischen der Justiz und der politischen Macht hauptsächlich in Europa auf. Während Italien der Vorreiter in Sachen politischer Spannungen war, die im Umfeld der Dritten Gewalt erzeugt wurden, haben andere Länder wie Belgien, Spanien, Polen, Frankreich oder Portugal mehr oder weniger regelmäßig wiederkehrende Verletzungen der Dritte Gewalt durch die politische und wirtschaftliche Macht (durch die Medien unterstützt) erlebt.

In Frankreich zum Beispiel wurde seit den neunziger Jahre vor dem Risiko des »Richterstaates« gewarnt. Das geschah, als die französischen Untersuchungsrichter sich erstaunt über die Flugtickets für Frau und Tochter des Präsidenten oder über die Lebensmittelausgaben des Elysée-Palastes zeigten. Die politischen Skandale in Paris während der neunziger Jahre führten dazu, ein »Komplott« in der Justiz gegen die demokratisch etablierte Macht zu unterstellen.

In Großbritannien, der Nation mit einem konsolidierten Justizsystem, das als das am meisten respektierteste der Welt erachtet wird, kann man im Jahre 2003 mit Erstaunen eine Feststellung des Innenministers zur Kenntnis nehmen. Der Kommentar war anlässlich eines Urteils abgegeben worden, das eine Entscheidung des Innenministeriums aufhob, die einer Immigrantengruppe Asyl verweigert hatte. Er sagte: »Ich wünsche mir, dass kein Zweifel zurückbleibt: wir akzeptieren die Entscheidung des Gerichts nicht. Ehrlich, ich habe genug von dieser Situation, in der das Parlament Entscheidungen trifft und die Richter seine Entscheidungen widerrufen«.

In Portugal hat die Diskussion über die Dritte Gewalt und ihre illegitimen Einflüsse, sich im letzten Jahrzehnt des 20. Jahrhunderts verändert. Zuerst bezog sich der Hauptkritikpunkt an der Justiz (billigerweise) auf die Verzögerungen und den Mangel an menschlichen Ressourcen sowie gesetzlichen und logistischen Mitteln, um eine effektive Justiz zu implementieren. Diese Art der Kritik führte die Meinungen von Bürgern und Justizexperten zusammen (Richtern, Staatsanwälten, Rechtsanwälten und anderen Staatsbeamten), die nicht nur gemeinsam ihren persönlichen Unmut vorbringen wollten, sondern die anerkannten, dass das System dringend Reformen benötigte. Die Spannungen zwischen politischer Macht und der Dritten Gewalt wurden erst durch die Lösungsvorschläge immer stärker, die jeweils von der anderen Seite abgelehnt wurden. Aus der Krise wegen fehlender Ressourcen wurde jedoch schnell eine politische und kulturelle Krise, die von 
der ersten völlig verschieden war. Derzeit geht es nicht nur um die zu lange Verfahrensdauer vor den erstinstanzlichen Gerichten einer Justiz, die idealerweise absolut und universal sein sollte, sondern hauptsächlich um die Aktion, Reaktion und Antwort einer Justiz, die nicht von politischen, wirtschaftlichen und sozialen Mächten gesteuert werden sollte, zumindest nicht direkt. Während in einem ersten Stadium die Analyse der Krise und mögliche Lösungen einstimmig und ohne Widerspruch in einem Wurf gelangen, ist derzeit ein Konsens über Lösungsvorschläge kaum noch herzustellen. Gegenwärtige unterschiedliche Interessen zeigen in Portugal und in vielen europäischen Ländern eine wirtschaftliche und technokratische Konzeption von Justiz, die mit dem Konzept vom neoliberalen Staat und einer in diesem Kontext verstandenen globalisierten Gesellschaft zusammenpassen. Die Gerichte werden als Institutionen im Sinne staatlicher, abhängiger Organismen verstanden, deren Entscheidungen ein Minimum an rechtlichen Schwankungen zeigen sollen. Die politischen und wirtschaftlichen Mächte, eine Gruppe von Rechtsanwälten und Teile der Justiz tragen zu dieser Konzeption weiter bei.

\section{Die Unabhängigkeit als Garant von Menschenrechten und Rechtsstaatlichkeit}

Dieser Konzeption muss man die Sicht der Justiz als zivilisatorischen Wert entgegenhalten, mitverantwortlich für die Herstellung sozialer Gerechtigkeit und der Grundpfeiler von Rechtsstaatlichkeit. Diese letztere Konzeption führt zur Existenz von unabhängigen und effektiven Gerichten. Reformen, die direkt oder indirekt die strukturgebenden Prinzipien der Dritten Gewalt berühren, sind bei dieser Konzeption nicht zulässig (Unabhängigkeit, Unabsetzbarkeit etc.).

Eine humanistische Konzeption der Justiz ist nicht vereinbar mit Produktivitätsindikatoren, mathematischen Gesetzen zur Bewertung der zu entscheidenden Tatsachen und noch weniger vereinbar mit Entscheidungen, die nur der Selbstzufriedenheit und höchstpersönlichen Maßstäben genügen. Die Justiz ist ein Wert der Menschheit und für die Menschheit. Die Richter müssen vor Augen haben, dass »jedermann von Unverletzlichkeit profitiert«, wie es John Rawls ausdrückte, »die durch die Justiz garantiert wird, die nicht beseitigt werden kann als ganzes, auch nicht zum Wohle der Gesellschaft«. Weiterhin müssen sie verstehen, dass »der Verlust der Freiheit einiger Bürger nicht zu rechtfertigen ist durch die Tatsache, dass andere Bürger eine größere Wohltat erfahren werden«; »sie dürfen nicht zulassen, dass manchen Bürgern Opfer abverlangt werden, die durch eine Vergrößerung der Vorteile zum Wohle der Mehrheit ausgeglichen und so gerechtfertigt werden«. Folglich können die von der Justiz garantierten Rechte nicht zur Disposition der Politik stehen oder von der Bewertung sozialer Interessen abhängig sein. Unrecht ist nur erträglich, wenn damit ein größeres Unrecht verhindert wird. Wenn in einer Gesellschaft wie der unsrigen die wahre Dimension von Recht und Justiz verloren geht, wird es möglich, alle Arten von Justizreformen durchzuführen im Namen von Produktivität, von größerem Profit oder geringerem Verlust, oder im Namen eines nicht an Werten oder Ethik orientierten Managements. In einem solchen Kontext wird die Aufgabe der Gerichte als Garant von Rechtsstaatlichkeit und Menschenrechten missverstanden, ist vielmehr unerwünscht und die Unabhängigkeit der Richter erscheint als ein abzuschaffender Mythos. 


\section{Die konfliktreiche Beziehung zwischen Politik und Richterschaft - Ein systemim- manentes Problem}

Die Spannungen in der Beziehung zwischen Politik und Richtern sind solchen Systemen inhärent, die die Rechtsprechung Einzelpersonen übertragen, die unabhängig von der politischen Macht sind: in den vergangenen absoluten Monarchien oder in den modernen Diktaturen hat es diese Probleme nicht gegeben. Wenn diejenigen, die Recht sprechen, unabhängige Einzelpersonen sind, ist ein Konflikt immer möglich; dies ist dem Konzept der Gewaltenteilung und der Unabhängigkeit der Richter immanent. Wenn der Konflikt zwischen den Gewalten stärker wird, reagieren die Politiker immer auf die gleiche Weise. Sie ändern das Gesetz dahingehend, dass sie die Unabhängigkeitsgarantien verringern und die Interventionsmöglichkeiten der Gerichte beschränken. Dies geschah in den USA unter Roosevelt, in Frankreich (z.B. unter De Gaulle und Mitterand); es passiert in Italien unter der Regierung Berlusconi; es passierte und es passiert in Portugal.

Anders als die Freiheit und Unparteilichkeit des Richters, die die psychologische und die ethisch-normative Dimension sowie das konkrete Verfahren betrifft, konstituiert die Unabhängigkeit die Autonomie des Richters vom politischen System. Die Vorstellungen, die man von Unabhängigkeit haben mag und ihrer Abstufung oder Konkretisierung, sind verbunden mit der konkreten Ausgestaltung des Rechtssystems, der Rechtsprechung und ihrer Rolle. Das Konzept von Unabhängigkeit ist sehr unterschiedlich in Ländern mit »common law « und Ländern mit »kodifiziertem Recht « und verändert sich auch je weiter man in Europa Richtung Norden und Süden blickt. Obwohl europäische Legislativen sich über die Nichtunterwerfung des Richters, außer unter Gesetz und Recht, einig sind, sind die Divergenzen offensichtlich, wenn man die Verfassungen der zahlreichen Justizsysteme in Europa vergleicht, besonders dann, wenn man ihr praktisches Funktionieren in die Betrachtung mit einbezieht. Die Unabhängigkeit mag eingeschränkt scheinen, wenn es um die Rekrutierung, Auswahl und Ernennung von Richtern, ihre Einstufung, Versetzung und Disziplinierung, und auch wenn es um Laufbahnbeförderung geht. Und sie mag auch noch auf eine subtilere Weise hinsichtlich der richterlichen Gesetzesauslegung und der exekutiven Umsetzung verkündeter Urteile beschränkt sein, was aber Richtern und ihren Verbänden weniger gewahr wird. Daneben trägt auch die Privatisierung bestimmter öffentlicher Bereiche - aus denen sich der Staat (aus wirtschaftlichen Gründen) langsam zurückzieht - dazu bei, dass die Gerichte ihrer Kontrollfunktion nicht mehr gerecht werden können.

Die interpretative Freiheit, also der Interpretationsspielraum bei der Gesetzesanwendung sowie richterliche Rechtsfortbildung, ist einer der interessantesten und komplexesten Aspekte der Beziehungen zwischen Politik und Justiz und der Definition von Unabhängigkeit der Justiz. Von Justinian bis Napoleon war es Richtern verboten, durch extensive Interpretationen den Gesetzestext zu entstellen oder Rechtsfortbildung zu betreiben, die nicht aus einer einfachen lückenfüllenden Kreativität entsteht, die im - durch die Legislative absichtlich oder unabsichtlich belassenen - Vakuum bei gesetzlich nicht geregelten Fällen entwickelt wird. Trotzdem ist es heute unmöglich, sich 
eine automatische und richtige Anwendung der gesetzlichen Normen vorzustellen, die per se allgemein verbindlich wirken, umso mehr, als die Gesetzgebung sich in alle Richtungen des sozialen Lebens ausdehnt, manchmal in partieller, mit Leerstellen behafteter und widersprüchlicher Weise. Das positivistische Prinzip des Richters als »bouche de la loi « gründet auf einem gänzlich fehlendem Realismus. Kein normatives System ist imstande, alles menschliche Verhalten vorauszusagen und auf dieses zu reagieren; es muss die Rechtsfortbildung durch die Gerichte hinzukommen. Die Rechtsfortbildung durch den Richter birgt das Risiko, wie jede andere kulturelle Aktivität, dass ausschließlich die subjektiven Maßstäbe des Interpreten Anwendung finden. Die Interpretation von Gesetzen ist kein neutraler, reiner, geschmack- und geruchloser Akt (dies gilt nur für destilliertes Wasser); sie resultiert immer aus einer Auswahl von Werten, die niemand umgehen kann. All dies gefährdet nicht die Unparteilichkeit des Richters: sobald die interpretative Entscheidung getroffen ist, wird sie unparteiisch auf alle analogen Situationen und auf alle Bürger angewandt (ungeachtet des Glaubens, der Rasse oder wirtschaftlicher und sozialer Bedingungen). Daher haben die Hindernisse, die der Erfüllung des Unabhängigkeitsprinzips (und der Autonomie der Staatsanwälte) entgegenstehen, kein anderes Ziel als die Entscheidung zu kontrollieren (die Entscheidung über die Anklage oder die Verfahrenseinstellung oder die richterliche Entscheidung).

Da es politisch nicht korrekt ist, in die richterliche Entscheidung - zumindest direkt - einzugreifen, gibt es mehrere gesetzliche Mechanismen und Verfahrensakte, um die Aktivität des Richters einzuschränken oder sie zu einer funktionalen bzw. effizienten Entscheidung zu zwingen. Die Richter sind unabhängig in ihren Entscheidungen - laut Gesetz und explizitem politischen Diskurs. Wirklich? Und wie steht es mit den Hindernissen aus verfahrensökonomischen Gründen? Und mit den Hindernissen in der Gerichtsorganisation? Und mit den Karrieren der Richter? Und der Trennung von richterlicher und administrativer Tätigkeit innerhalb der Justiz mancher Länder? Und der Rechenschaftspflicht des Richters? Und der inadäquaten oder nicht vorhandenen Grund- und Weiterbildung? Und mit Zusammensetzung und Rolle des Kontrollgremiums für die Justiz? Und mit der fehlenden Durchsetzung richterlicher Entscheidungen? Und der Umgehung der Justiz durch Bereitstellung anderer Verfahren und Methoden? Und das fortgesetzte illegitime Vorgehen der Medien, mit der Zustimmung oder dem Schweigen der politischen Macht?

Die Unabhängigkeit der Dritten Gewalt soll in Portugal und in den meisten Ländern mit »kodifiziertem Recht« auf die höchste Ebene - die Verfassung - bezogen sein. Auf europäischer Ebene hat es mehrere Versuche gegeben, um dies sicherzustellen. Auch hier werden wieder die Unterschiede zwischen Ländern mit einer professionellen Justiz (common law) und Ländern mit bürokratischer Justiz (dem größeren Teil der Länder mit »kodifiziertem Recht«) sichtbar.

\section{Europäische Bemühungen zur Stärkung der Unabhängigkeit der Dritten Gewalt}

Der Europarat entwickelt, mit seinen zahlreichen Kommissionen, wie dem »Consultative Council of the European Judges« (CCJE) ein theoretisches Konzept mit Umset- 
zungsprogramm, um die Unabhängigkeit der Judikative und besonders die der Richter zu wahren. Es war kein Zufall, dass die erste »Stellungnahme« des CCJE der Unabhängigkeit der Dritten Gewalt gewidmet war. Sie stellt fest, dass die Unabhängigkeit der Richter eine Grundvoraussetzung von Rechtsstaatlichkeit und eine wesentliche Garantie für einen fairen Prozess ist. Die Unabhängigkeit ist weit davon entfernt, ein Vorrecht oder Privileg der Richter zu sein. Sie ist eine Garantie, die den Bürgern gegeben wird. Die Unabhängigkeit ist eine Garantie für (objektive) Unparteilichkeit wie die Unparteilichkeit eine Garantie für die Neutralität (subjektive Unparteilichkeit) der Richter ist.

\section{Neutralität}

Die Neutralität ist die wichtigste Eigenschaft des fairen Richters. Eine Person ist nicht fair, weil sie Richter ist, sondern sie ist Richter, um fair zu sein. Und um fair zu sein, muss man neutral sein. Neutral sein bedeutet, nicht betroffen zu sein: durch seine Vorurteile, seine Hassobjekte, seine Leidenschaften, seine Vorlieben und Abneigungen; als menschliches Wesen hat der Richter Schwierigkeiten, sie zu vermeiden. Weiterhin muss er der Gemeinschaft oder Gesellschaft, Vereinigungen oder Gruppen, die Druck ausüben, neutral gegenüberstehen. Die Neutralität gründet strukturell auf der Bindung des Richters an Gesetz und Recht, ergänzt durch ein klares Streben nach und ein starkes Gefühl für Gerechtigkeit. Neutralität ist jedoch nicht zu verwechseln mit Unparteilichkeit und Unabhängigkeit. Die Unparteilichkeit sichert die Neutralität wie Unabhängigkeit die Unparteilichkeit sichert. Man ist unparteilich, um neutral zu sein und unabhängig, um unparteilich zu sein. Während die Neutralität eine persönliche Eigenschaft (und Pflicht) eines Richters ist, ist die Unparteilichkeit eine Verfahrensbedingung, und die Unabhängigkeit ist ein grundlegendes Prinzip, das richterliche Gewalt an Rechtsstaatlichkeit koppelt.

\section{Unparteilichkeit}

Unparteilich zu sein bedeutet, nicht in die Belange der Parteien verwickelt zu sein. Die Unparteilichkeit bedeutet, dass der Richter allein dem Gesetz unterstellt ist. Sie ist Quelle der richterlichen Entscheidung und Voraussetzung ihrer politischen Legitimierung und bedeutet, dass der Richter nicht in Ansehung der Person oder der politischen Umstände entscheiden darf. Der Richter ist weder im besonderen noch im allgemeinen daran interessiert, eine bestimmte Lösung für die Kontroverse zu finden, die zu lösen er aufgerufen ist, da seine Funktion darin besteht, zu entscheiden, was wahr und was unwahr ist, richtig oder falsch, legal oder illegal. Keinerlei Interesse oder Wunsch soll sein Urteil beeinflussen.

\section{Unabhängigkeit}

Die Unparteilichkeit erfordert eine institutionelle Trennung des Richters von den Parteien, während die Unabhängigkeit die institutionelle Trennung der richterlichen Gewalt von den übrigen Staatsgewalten erfordert. Im Unterschied zur Legislative oder der Exekutive, die Mehrheitsgewalten sind, spricht der Richter Recht im Namen des 
Volkes, aber nicht im Namen der Mehrheit und hat die Rechte der Minderheiten zu verteidigen. Die Unabhängigkeit führt einerseits zur institutionellen Trennung der Dritten Gewalt als solcher und der Trennung der einzelnen Richter von den übrigen Staatsgewalten (äußere Unabhängigkeit) sowie andererseits zur Freistellung von seinen psychologisch bedingten Vorurteilen (innere Unabhängigkeit). Dies ist notwendig für die Garantie von Unparteilichkeit und folglich für die Garantie von Gleichbehandlung der Bürger vor dem Gesetz. Unabhängigkeit und Unparteilichkeit sind die kennzeichnenden Elemente eines triadischen richterlichen Prozesses, der durch die Gegenwart eines Richters bestimmt wird.

Die Unabhängigkeit der Dritten Gewalt abgeleitet aus der Gewaltenteilung darf nicht nur eine theoretische Unabhängigkeit bleiben. Sie muss real und in vollem Umfang in der Justiz und ihrer Organisation existieren, um dem Rechtssystem die operationelle Basis zur Erfüllung seiner Aufgabe in der Gesellschaft zu schaffen. Das bedeutet, dass die archaischen Strukturen eines exekutiven Paternalismus aufgebrochen werden müssen, die im Umgang mit Richtern vorhanden sind. Die Unabhängigkeit der Dritten Gewalt muss funktional und ökonomisch sein. Diese Unabhängigkeit der Richter wird verkörpert durch die Existenz eines Verwaltungsorgans der Justiz, dem Justizverwaltungsrat, und durch die Existenz möglicher anderer Organe (Gerichtsgremien) zur Regelung der Gerichtsverwaltung, die landesspezifische Unterschiede aufweisen können. Ferner wird Unabhängigkeit durch die nicht-hierarchische Aufstellung von Richtern und durch das Fehlen administrativer Steuerungsvorschriften innerhalb des Justizsystems gewährleistet. Richter haben keine Anordnungen oder Anweisungen zu erhalten - außer auf dem Wege eingelegter Rechtsmittel.

\section{Die Empfehlungen des Beirats der Europäischen Richter (CCJE)}

Der CCJE hat nicht nur die Grundsätze definiert, die die Unabhängigkeit sicherstellen. Die folgenden »Stellungnahmen« nehmen die praktische Anwendung des Unabhängigkeitskonzepts in den Blick. Mit der Umsetzung grundlegender Prinzipien wie Unabsetzbarkeit oder der Ernennung auf Lebenszeit entsteht eine effektive, nicht-abhängige richterliche Organisation. Dazu gehört ebenfalls eine klare Definition der Laufbahn des Richters, die durch objektive Auswahlkriterien geleitet werden soll. Solche wären ihre Verdienste, ihre Bewertungen, ihre Integrität, ihre Kompetenz und ihre Effektivität. Trotzdem ist die Unabhängigkeit eng verbunden mit der anfänglichen Aus- und Weiterbildung der Richter (Stellungnahme Nr. 4 des CCJE) wie auch mit ihrer Ethik und Zuverlässigkeit (Stellungnahme Nr. 3 des CCJE).

Die Schaffung des so genannten Justizverwaltungsrats wurde durch die Stellungnahme des CCJE besonders in den Mittelpunkt gerückt (Stellungnahme Nr. 10). Der wichtigste Aspekt der verfassungsmäßigen Garantie der Unabhängigkeit der Richter besteht in dem Verbot der Exekutive, die Richter aus ihrem Amt wegzuversetzen oder sie ihres Amtes zu entheben als Vergeltung für von ihnen verkündete unangenehme Entscheidungen, also der Grundsatz der Ernennung auf Lebenszeit und der Unabsetzbarkeit. Dieser Grundsatz ist auch typisch für die englische Tradition seit 1700 und wurde auch in die amerikanische Verfassung aufgenommen. Diese wesentliche Garan- 
tie ist ein Merkmal der Systeme des »common law «, in denen die Justiz nicht-bürokratisch und nicht-hierarchisch aufgebaut ist. Gewöhnlich führt der Richter die Funktionen fort, für die er ernannt wurde.

In Systemen, in denen die Justiz in stärkerer Weise bürokratisiert ist, in denen junge Richter (nach ihrem Hochschulstudium) Zugang zu einem Laufbahnsystem innerhalb einer hierarchischen Organisation haben, spielt das Prinzip der Unabsetzbarkeit die vorrangige Rolle. Die Unabsetzbarkeit verhindert die zufällige Versetzung und sichert die Unabhängigkeit richterlicher Anordnungen, vor allem aber die Unabhängigkeit jedes Richters. Wenn die Laufbahn jedoch durch Aufstieg und Vergütung bestimmt wird, ist die Garantie der Unabsetzbarkeit nicht ausreichend. Um den Anforderungen der Autonomie der Dritten Gewalt und der richterlichen Unabhängigkeit zu genügen, wurde die moderne Idee des Justizverwaltungsrats geboren. In einer hierarchisch strukturierten Justiz sind die klassischen Garantien, wie die der Unabsetzbarkeit, einer Garantie von rein statischer Unabhängigkeit, nicht ausreichend. Es müssen dynamische Garantien geschaffen werden, die die richterlichen Laufbahnen der Kontrolle der Exekutive und hierarchischen Einflüssen entziehen.

Die Idee eines Organs wie die des Justizverwaltungsrats ist nicht neu. Sie entstand ursprünglich in Frankreich und Italien und wurde in Portugal und Spanien nach dem Ende der Diktaturen und in Mittel- und Osteuropa nach dem Fall des Kommunismus entwickelt. In Portugal, Italien und Spanien hat die Erfahrung der Diktatur zu einem kollektiven Bewusstsein der Richter über die Notwendigkeit einer selbstverwalteten Justiz als Garantie der Unabhängigkeit beigetragen. Der CCJE unterstützt prinzipiell keine korporative Zusammensetzung des Justizverwaltungsrats. Eine gemischte Zusammensetzung jedoch bedeutet, dass der demokratische Staat die Notwendigkeit erkannt hätte, der Justiz ein Managementsystem zur Verfügung zu stellen, das eine Übermacht der Richter verhindert, die Koordination mit der Volksvertretung fördert und die Garantie von Unabhängigkeit und Nicht-Manipulation durch diese gemischte Zusammensetzung des Justizverwaltungsrats sicherstellt. Obwohl Europa bis heute noch kein standardisiertes Modell eines Justizverwaltungsrats besitzt, sind doch allgemeine Merkmale zu erkennen, die die Modelle einander näher bringen. Seine Rolle, Eigenschaften und Zusammensetzung sind von grundlegender Bedeutung.

Was die Zusammensetzung angeht, so ist die Tendenz mehrerer Kommissionen des Europarates, die sich mit der Unabhängigkeit der Judikative befassen, die, einen gemischten Justizverwaltungsrats zu schaffen, der aus Richtern von Gerichten unterschiedlicher Instanz und aus Laienmitgliedern besteht. Er soll nicht ausschließlich aus Richtern bestehen (oder Richtern von Höheren Gerichten), sondern er soll die unterschiedlichen Meinungstrends sowohl der Justiz wie der Gesellschaft widerspiegeln, und so die Legitimationsquelle der Dritten Gewalt darstellen. Auf der anderen Seite soll er aus einer Mehrheit von Richtern bestehen, die von ihren Kollegen gewählt werden. So könnte politische Manipulation oder jeglicher unangemessener Druck vermieden werden. Das Organ soll seine Funktion mit einem Minimum an Zugeständnissen wegen wechselnder Parlamentsmehrheiten ausüben können und ohne jegliche implizite Unterordnung unter parteigängerische Logik bestehen, um Garant essentieller Werte und Prinzipien sein zu können. Der geforderte Korporatismus befindet sich im 
Widerspruch zur pluralistischen Vertretung der Justiz durch den Justizverwaltungsrat und zur externen Kontrolle dieses Organs. Die Existenz des Justizverwaltungsrats gründet auf der Garantie von Unabhängigkeit und Autonomie der Judikative und insbesondere der Richter. Daher soll er neben den Verwaltungs-, den Management- und den Disziplinarfunktionen der Dritten Gewalt die essentiellen Werte garantieren: die Unabhängigkeit, den richterlichen Pluralismus, den Schutz der Dritten Gewalt als Bewahrerin von Freiheit und Menschenrechten und die Qualität der ständigen Diskussion über die Rolle der Judikative innerhalb eines demokratischen Systems.

Wir sprechen über Unabhängigkeit der Dritten Gewalt; dennoch sind wir gegenwärtig Zeugen eines (natürlichen oder erzeugten) wachsenden Misstrauens der Bürger gegenüber ihren Richtern. Es gibt viele Gründe für das Misstrauen: hauptsächlich diejenigen kultureller Art und diejenigen, die mit der Tätigkeit des richterlichen Apparats verbunden sind. Die Unabhängigkeit ist nur sichtbar und zu verstehen, wenn die richterliche Aktivität mit Professionalität und in angemessener Zeit durchgeführt wird. 